\title{
Calidad de vida y actividades de la vida diaria en adultos mayores mexicanos
}

\author{
Quality of life and daily life activities \\ in Mexican older adults
}

\author{
Alejandro Pérez Ortizin, Ana Leticia Becerra Gálvez², \\ Guadalupe Arely Hernández Gálvez ${ }^{1}$, María del Carmen Estrada Alvarado ${ }^{1}$ \\ y Erick Alberto Medina Jiménez ${ }^{l}$
}

Universidad Nacional Autónoma de México ${ }^{1,2}$

Autor de correspondencia: Alejandro Pérez Ortiz, psicaleperez14@gmail.com.

\begin{abstract}
RESUMEN
Las personas de 60 años y más experimentan una serie de complicaciones biopsicosociales que pueden afectar la ejecución de actividades de la vida diaria y posiblemente con ello su independencia, autoeficacia y calidad de vida, no obstante, poco se conoce si dichas variables realmente están asociadas. El objetivo de esta investigación fue identificar la asociación entre calidad de vida y autoeficacia para realizar actividades cotidianas, así como con otras variables de índole sociodemográfico en un grupo de adultos mayores mexicanos; 102 participantes contestaron el Cuestionario de Detección de Salud en la Vejez (ex profeso), el Cuestionario de Calidad de Vida para Adultos Mayores (WHOQOL-OLD) y el instrumento de Autoeficacia para Realizar Actividades Cotidianas (AeRAC-Breve). Los hombres presentaron mejor calidad de vida $(\mathrm{U}=.036, p<.05)$; se obtuvo una correlación positiva de magnitud pequeña entre el WHOQOL-OLD y el AeRAC-Breve $(r=.363, p<.000)$. Las correlaciones más altas se encontraron en la dimensión tres de ambos instrumentos (intimidad y relaciones sociales; $r=.768, p<.001$ ) y la seis del WHOQOL-OLD con la dimensión tres del AeRAC-Breve $(r=.855, p<.001)$. Poco más de la mitad de la muestra presentó niveles intermedios y bajos en el WHOQOL-OLD y AeRAC-Breve. Continuar con el estudio de estas variables (calidad de vida y actividades de la vida diaria) permitirá la formulación de un modelo teórico sólido, que a su vez dé paso al diseño de intervenciones psicológicas dirigidas a la población envejecida.
\end{abstract}

Palabras clave: Psicología de la salud; Psicología del Envejecimiento; Calidad de Vida; Adultos mayores.

\begin{abstract}
People aged 60 and over tend to experience biopsychosocial complications that may affect performing daily activities and compromising their independence, self-efficacy, and quality of life. However, little is known of the extent to which these variables associate for Mexican participants. The purpose of this study was to identify the association between quality of life, self-efficacy in daily activities, and sociodemographic variables in a group of Mexican older adults. A total of 102 participants answered an Old Age Health Screening Questionnaire, the Quality of Life Questionnaire
\end{abstract}

\footnotetext{
${ }^{1}$ Facultad de Estudios Superiores Iztacala, Av. De los Barrios s/n, Col. Los Reyes Iztacala, 54090 Tlalnepantla, Edo. de México, México, tel. (55)56-23-13-33, ext.39737, correos electrónicos: psicaleperez14@gmail.com, arelehernandez@gmail.com, carmen.mcea@gmail.com y erickdearco@gmail.com.

${ }^{2}$ Facultad de Estudios Superiores Zaragoza, Av. Guelatao 66, Ejército de Oriente Indeco II IsssTe, Iztapalapa, 09230 Ciudad de México, CDMX, correo electrónico: behaviormed.ana@gmail.com.
} 
for The Elderly (WHOQOL-OLD), and a Self-Efficacy instrument on Everyday Activities (AeRAC-Brief). Men showed better quality of life $(U=.036, p<.05)$; and modest positive correlation resulted between WHOQOL-OLD and AeRAC-Brief $(r=.363, p<.000)$. The highest correlations were found in intimacy and social relations in both instruments; $r=7.68$, $p<.001)$ and dimension six of WHOQOL-OLD with the dimension three of AeRAC-Brief ( $r=.855$, $p<.001)$. Just over half of the sample showed intermediate and low levels in WHOQOL-OLD and AeRAC-Brief. Results point toward the need to study these variables further to formulate a more solid theoretical model, which will, in turn, lead to the design of psychological interventions aimed at the elderly population.

Key words: Health Psychology; Psychology of aging; Quality of life; Old adults.

Recibido: 29/08/2020.

Aceptado: 18/01/2021.

A finales del siglo XX se suscitaron en todo el mundo diversas cuestiones relativas a la salud y a la educación, entre muchas más; como una consecuencia indirecta de lo anterior, la esperanza de vida aumentó, por lo que hubo un aumento importante de la población mayor de 60 años. Hasta 2019 se habían registrado 703 millones de personas con una edad igual o mayor a 65 años, y las proyecciones demográficas para el año 2050 estiman que este grupo etario alcanzará los 1.5 miles de millones (Castillo y Vela, 2005; United Nations Organization, 2019). En el caso de América Latina han ocurrido cambios demográficos de importancia ya que han disminuido la mortalidad y la fecundidad. México es uno de los principales países de dicha región que enfrenta un proceso de envejecimiento poblacional acelerado, por lo que se estima que para 2050 este grupo constituya cerca de $19.9 \%$ de la población total (González, 2016; Instituto Nacional de las Personas Adultas Mayores [INAPAM], 2019; Lesson, 2013).

Una mayor esperanza de vida y un comportamiento poco saludable aumentan la probabilidad de desarrollar una enfermedad crónico-degenera- tiva. En el mundo, los adultos mayores (AM, en lo sucesivo) suelen padecer enfermedades cardiovasculares, diabetes, cáncer, enfermedades pulmonares crónicas, enfermedades músculo-esqueléticas, trastornos mentales y enfermedades del sistema nervioso -sin importar su edad o funcionalidad-, aunado a distintas discapacidades, por lo que incluso sufren discriminación y desigualdad (Consejo para Prevenir y Eliminar la Discriminación de la Ciudad de México, 2016; Huenchuan, 2013; Instituto Nacional de las Mujeres, 2015; Montes de Oca, 2013).

En consecuencia, el riesgo de padecer una enfermedad crónica, aunado a las condiciones físicas y sociales que afrontan los AM, pueden afectar su calidad de vida ( $\mathrm{CV}$, en lo sucesivo) y su bienestar. De acuerdo con la Organización Mundial de la Salud (OMS) (1996), la CV se define como "la manera en la que el individuo percibe el lugar que ocupa en el entorno cultural y en el sistema de valores en que vive, así como en relación con sus objetivos, expectativas, criterios y preocupaciones" (p. 385). Así pues, incluye la salud biopsicosocial, la independencia, las relaciones sociales, los factores ambientales y las creencias personales. Algunos de los elementos identificados que pueden mermar la CV en los AM latinoamericanos son el ser mujer, ser analfabeto, padecer una enfermedad crónico-degenerativa, tener bajos ingresos económicos, ejercer una ocupación u oficio modesto, sufrir depresión, ansiedad o deterioro cognitivo, disponer de una red social limitada, percibir su salud como "mala", padecer maltrato por parte de familiares, y ver su independencia limitada (Cardona, Segura, Garzón y Salazar, 2018; Estrada et al., 2011; Soria y Montoya, 2017; Villegas y Montoya, 2014).

Por el contrario, la percepción de una alta o "buena" CV en este grupo etario se ha asociado con tener independencia financiera, contar con casa propia, vivir en compañía, gozar de cuidados y disponer de una red social amplia y funcional (Garbaccio, Tonaco, Estêvão y Barcelos, 2018; Granados y Muñoz, 2015).

Como se señaló anteriormente, se ha propuesto la independencia como una dimensión de la CV que involucra la movilidad y las actividades de la vida diaria (AVD en adelante). Las AVD son 
aquellas conductas que una persona lleva a cabo para poder cuidar de sí misma con independencia y autonomía (Romero, 2007). De acuerdo a Martínez, Ibarrola, Fernández y Lafita (2017), el término más apropiado al hablar de "independencia" es capacidad funcional, que comprende los atributos relacionados con la salud que le permiten a una persona ser y hacer lo que es importante para ella, y se compone de la capacidad intrínseca, las características del entorno que afectan a esta y las interacciones con otros; no obstante, la capacidad funcional se evalúa cuantitativamente, es decir, qué puede hacer o no hacer un individuo por sí mismo. Siguiendo esta propuesta, la capacidad funcional de un AM será deficiente si este manifiesta limitaciones físicas, o bien si su entorno no le permite llevarlas a cabo. Es por ello que la autoeficacia, entendida como la valoración que hacen los individuos sobre su capacidad o su competencia para ejecutar con éxito una conducta específica hará posible evaluar el componente subjetivo en la capacidad funcional, ampliar el paradigma en la conceptuación y evaluación del mismo, y promover un envejecimiento saludable (Bandura, 1997; Grembowski et al., 1993; OMS, 2015).

La evidencia que aporta la literatura especializada entre la capacidad funcional o la autoeficacia en la ejecución de AVD y la relación con la CV en los AM es muy escasa, por lo que analizar ambas variables permitirá conocer su relación, para luego poder diseñar intervenciones psicológicas que mejoren la CV y las AVD de los AM. Además, en la percepción de autoeficacia, funcionalidad y CV del AM convergen otras variables propias del contexto sociocultural que podrían ser disposicionales para esta última; por ejemplo, el estado civil (v.g., solteros o viudos) (Montes de Oca, 2013), el nivel socioeconómico (Cardona et al., 2018, Garbaccio et al., 2018), las redes de apoyo (Garbaccio et al., 2018) y la seguridad social, y el acceso a los servicios de salud (Villegas y Montoya, 2014). Con base en lo anteriormente expuesto, el objetivo de la presente investigación fue identificar la asociación entre la CV y la autoeficacia para llevar a cabo las actividades cotidianas, así como con otras variables sociodemográficas en un grupo de AM mexicanos.

\section{MÉTODO}

\section{Participantes}

Se utilizó una muestra no probabilística de tipo intencional conformada por adultos mayores de 60 años, quienes fueron reclutados en diversos espacios recreativos, educativos y en centros del Sistema Nacional para el Desarrollo Integral de la Familia. El criterio de inclusión fue tener 60 años o más, en tanto que el de eliminación fue responder menos del $80 \%$ de los instrumentos de medición.

\section{Instrumentos}

Cuestionario de detección de salud en la vejez.

Dicho cuestionario fue elaborado ex profeso por los presentes autores y se compone de tres secciones: datos sociodemográficos (edad, sexo, estado civil, escolaridad, y ocupación); nivel socioeconómico (ingresos mensuales y preguntas para la detección de dicho nivel, elaboradas por la Asociación de Agencias de Inteligencia de Mercado y Opinión Pública, A.C. [2017)), y estado de salud y seguro médico (que considera la afiliación a un seguro médico, patologías presentadas, años de padecerlas y atención de las mismas).

\section{Cuestionario de Calidad de Vida para Adultos Mayores (WHOQOL-OLD).}

Este instrumento fue elaborado por Power, Quinn, Schmidt y el WHOQOL-OLD Group (2005), y validado y adaptado a población mexicana por González y Gómez (2013). Posee una varianza explicada de $65.70 \%$ y un coeficiente alfa de Cronbach alto $(\alpha=0.83)$. Se compone de seis factores: Actividades (pasadas, presentes y futuras), Autonomía, Intimidad, Participación social, Muerte y Morir, evaluados con 24 reactivos con cinco opciones de respuesta en una escala tipo Likert (de 1: "Nada", a 5: "Completamente). La puntuación mínima es 24 y la máxima 120, donde a mayor puntuación, mayor calidad de vida.

\section{Instrumento de Autoeficacia para Realizar} Actividades Cotidianas (AeRAC-Breve).

Diseñado por González (2009), posee una varianza explicada de $62.417 \%$ y un coeficiente alfa de 
Cronbach alto $(\alpha=0.87)$. Está compuesto por cinco factores: Actividades de independencia, Actividades recreativas mentales, Actividades sociales, Actividades de protección a la salud y Actividades recreativas físicas, evaluados mediante 26 reactivos con cinco opciones de respuesta en una escala tipo Likert: "Nada" $=0$; Un poco $=25$; Moderadamente $=50 ;$ Bastante $=75$, y Completamente $=100$. A mayor puntuación, mayor autoeficacia percibe el AM para realizar sus actividades cotidianas.

Para facilitar el análisis entre los instrumentos empleados se efectuó lo propuesto por González y Lima (2017) y González y Raya (2017), esto es, transformar los puntajes brutos en el WHOQoL-OLD y el AeRAC-Breve en una escala de 0 a 100 siguiendo la siguiente fórmula:

$$
\text { WHOQOL - OLD100 }=\frac{(X-24)(100)}{96}
$$

Donde:

WHOQOL-OLD $100=$ Puntuación bruta total obtenida en el instrumento y transformada a una escala de 0 a 100.

Para el WHOQoL-OLD se obtuvo la diferencia de -24 a cada puntuación, para iniciar el rango desde 0 hasta $120-24=96$.

$\mathrm{x}=$ Puntuación bruta total obtenida en el instrumento.

Para el AeRAC-Breve se obtuvo la puntuación bruta total y se empleó la siguiente fórmula:

$$
\text { AERAC }- \text { BREVE }=\frac{(x)(100)}{2600}
$$

Donde:

AeRAC-Breve $=$ Puntuación bruta total obtenida en el instrumento y transformada a una escala de 0 a 100.

$\mathrm{x}=$ Puntuación bruta total obtenida en el instrumento.

\section{Procedimiento}

Los AM que decidieron participar lo hicieron de manera voluntaria, para lo cual leyeron y firmaron un formato de consentimiento informado. La aplicación de los instrumentos se hizo de manera presencial en un formato grupal; así, las preguntas y opciones de respuesta se les leían en voz alta, y te-

Instituto de Investigaciones Psicológicas - Universidad Veracruzana nían unos segundos para responder, para después resolver dudas en caso de haberlas. Se utilizó un formato individual para aquellos participantes analfabetos o que tuvieran dificultades para comprender las preguntas o las opciones de respuesta; en ambos casos, se realizó el mismo proceso, esto es, lectura de preguntas y opciones de pregunta en voz alta, y resolución de dudas si es que las había. En cada aplicación se encontraban en el lugar al menos tres de los investigadores a fin de asistir individualmente a los participantes que lo requirieran.

El riesgo de participar en la investigación fue mínimo ya que se trató de un estudio con alcance descriptivo-transversal cuyo fin fue recabar información de índole psicosocial y sin emplear procedimientos psicoterapéuticos. Se procuró la transparencia de los datos proporcionando información a los participantes sobre el proyecto, sus responsables y los medios de contacto en caso de que tuviesen dudas. En el cuestionario se indicó que el tratamiento de los datos personales sería confidencial y solamente con fines de investigación científica. En cada espacio donde se efectuó la aplicación se contó con la aprobación verbal y escrita de las autoridades responsables.

\section{Análisis de datos}

Los resultados obtenidos se analizaron empleando el SPSS, versión 20. Se obtuvieron los análisis estadísticos descriptivos de la muestra y se realizó la prueba de bondad de ajuste, obteniendo como resultado que las variables no tenían una distribución normal; en consecuencia, se optó por realizar pruebas no paramétricas. Para conocer las diferencias entre la CV y las AVD se utilizó la prueba $U$ de Mann-Whitney, y para evaluar el grado de asociación entre las variables, el coeficiente $r$ de Pearson. La significancia estadística se determinó con un nivel de $p \leq .05$.

\section{RESULTADOS}

\section{Análisis descriptivo}

La muestra se conformó por 102 adultos mayores (86 mujeres y 16 hombres), de entre 60 y 95 años 
( $\mathrm{M}=69$ años). En cuanto al nivel educativo, casi la mitad de los participantes contaban con educación básica; por otro lado, los participantes reportaron contar con ingresos económicos mensuales de entre $\$ 800.00$ y $\$ 50,000(\mathrm{M}=\$ 5,350.00)$.
Solo un participante no sabía leer ni escribir. La Tabla 1 muestra las características de los participantes en términos de su frecuencia y porcentaje.

A su vez, la Tabla 2 muestra los niveles obtenidos de CV y AVD por los participantes.

Tabla 1. Características de los participantes.

\begin{tabular}{|c|c|c|c|c|c|}
\hline Característica & $f$ & $\%$ & Característica & $f$ & $\%$ \\
\hline \multicolumn{3}{|c|}{ Sabe leer y escribir } & \multicolumn{3}{|l|}{ Ocupación } \\
\hline Sí & 101 & 99.02 & Hogar & 46 & 45.0 \\
\hline No & 1 & 0.98 & Pensionado & 37 & 36.3 \\
\hline \multicolumn{3}{|l|}{ Escolaridad } & Profesional & 6 & 5.9 \\
\hline Primaria & 31 & 30.3 & Otro & 5 & 4.9 \\
\hline Secundaria & 20 & 19.6 & Empleado general & 3 & 2.9 \\
\hline Preparatoria & 20 & 19.6 & Comerciante & 2 & 2.0 \\
\hline Universidad & 21 & 20.6 & No contestó & 2 & 2.0 \\
\hline Posgrado & 1 & 1.0 & Oficio & 1 & 1.0 \\
\hline Sin instrucción & 7 & 6.9 & \multicolumn{3}{|c|}{ Afiliación a seguro médico } \\
\hline No contestó & 2 & 2.0 & IMSS & 64 & 62.7 \\
\hline \multicolumn{3}{|l|}{ Estado civil } & ISSSTE & 22 & 21.5 \\
\hline Viudo & 39 & 38.2 & Seguro popular & 5 & 4.9 \\
\hline Casado & 27 & 26.5 & No tiene & 5 & 4.9 \\
\hline Soltero & 18 & 17.6 & PEMEX & 2 & 2.0 \\
\hline Separado & 12 & 11.8 & No contestó & 2 & 2.0 \\
\hline Unión libre & 4 & 3.9 & ISSEMYM & 1 & 1.0 \\
\hline \multicolumn{3}{|c|}{ Nivel socioeconómico (AMAI) } & Otro & 1 & 1.0 \\
\hline $\mathrm{A} / \mathrm{B}$ & 54 & 52.9 & \multicolumn{3}{|l|}{ Enfermedades } \\
\hline $\mathrm{C}+$ & 15 & 14.7 & Diabetes mellitus & 30 & 29.4 \\
\hline $\mathrm{C}-$ & 11 & 10.8 & Hipertensión arterial & 29 & 28.4 \\
\hline $\mathrm{D}^{+}$ & 1 & 1.0 & Ninguna & 18 & 17.7 \\
\hline $\mathrm{D}$ & 1 & 1.0 & No contestó & 10 & 9.8 \\
\hline $\mathrm{E}$ & 0 & 0 & Otra & 9 & 8.8 \\
\hline No contestó & 20 & 19.6 & Cardiovasculares & 4 & 3.9 \\
\hline & & & Obesidad & 1 & 1.0 \\
\hline & & & Prediabetes & 1 & 1.0 \\
\hline
\end{tabular}

Nota $:$ AMAI $=$ Asociación de Agencias de Inteligencia de Mercado y Opinión Pública. IMSS = Instituto Mexicano del Seguro Social; ISSSTE $=$ Instituto de Seguridad Social al Servicio de los Trabajadores del Estado. PEMEX = Petróleos Mexicanos. ISSEMyM = Instituto de Seguridad Social del Estado de México y Municipios. 
Tabla 2. Niveles de CV y AeRAC en función del sexo.

\begin{tabular}{|c|l|c|c|l|c|c|}
\hline Sexo & Nivel de CV & $\boldsymbol{f}$ & $\mathbf{\%}$ & Nivel de AVD & $\boldsymbol{f}$ & $\mathbf{\%}$ \\
\hline \multirow{4}{*}{ Mujer } & Mala & 23 & 26.7 & Mala & 17 & 19.8 \\
\cline { 2 - 7 } & Regular & 21 & 24.4 & Regular & 22 & 25.6 \\
\cline { 2 - 7 } & Buena & 22 & 25.6 & Buena & 22 & 25.6 \\
\cline { 2 - 7 } & Muy buena & 20 & 23.3 & Muy buena & 25 & 29.0 \\
\hline \multirow{5}{*}{ Hombre } & Mala & 1 & 6.3 & Mala & 6 & 37.5 \\
\cline { 2 - 7 } & Regular & 4 & 25.0 & Regular & 3 & 18.7 \\
\cline { 2 - 7 } & Buena & 3 & 18.7 & Buena & 2 & 12.5 \\
\cline { 2 - 7 } & Muy Buena & 8 & 50.0 & Muy Buena & 5 & 31.3 \\
\hline
\end{tabular}

Nota $:$ CV $=$ Calidad de Vida; $\mathrm{AVD}=$ Actividades de la Vida Diaria.

\section{Análisis de correlación}

Se realizó la asociación entre las variables CV y AVD, obteniéndose una correlación positiva de magnitud leve $(r=.363, p<.000)$. Por otro lado, al realizar la asociación entre las dimensiones de los instrumentos WHOQOL-OLD y AeRAC-Breve se en- contraron trece coeficientes de correlación significativos de débiles a considerables. Los dos coeficientes de mayor magnitud se obtuvieron entre la tercera dimensión de ambos instrumentos $(r=.768$, $p<.001)$ y la sexta del WHOQOL-OLD con la tercera dimensión tres del AeRAC-Breve $(r=.855$, $p<.001$ ) (Tabla 3).

Tabla 3. Coeficientes de correlación entre las dimensiones del WHOQOL-OLD y las del AeRAC-Breve.

\begin{tabular}{|c|c|c|c|c|c|c|}
\hline \multirow{8}{*}{$\begin{array}{l}0 \\
0 \\
1 \\
1 \\
0 \\
0 \\
0 \\
0 \\
0\end{array}$} & \multicolumn{6}{|c|}{ AeRAC-Breve } \\
\hline & Factores & $\begin{array}{c}\text { Actividades de } \\
\text { independencia (1) }\end{array}$ & $\begin{array}{l}\text { Recreativas } \\
\text { mentales (2) }\end{array}$ & $\begin{array}{l}\text { Actividades } \\
\text { sociales (3) }\end{array}$ & $\begin{array}{l}\text { Protección } \\
\text { a la salud (4) }\end{array}$ & $\begin{array}{c}\text { Recreativas } \\
\text { físicas (5) }\end{array}$ \\
\hline & $\begin{array}{l}\text { Actividades pasadas } \\
\text { presentes y futuras (1) }\end{array}$ & n.s. & n.s. & $\begin{array}{c}r=.409 * * \\
p=.000\end{array}$ & $\begin{array}{c}r=.255^{* *} \\
p=.010\end{array}$ & n.s. \\
\hline & Autonomía (2) & n.s. & n.s. & n.s. & n.s. & n.s. \\
\hline & Intimidad (3) & $\begin{array}{c}r=.281^{* *} \\
p=.004\end{array}$ & n.s. & $\begin{array}{c}r=.768^{* *} \\
p=.000\end{array}$ & $\begin{array}{c}r=.295^{* *} \\
p=.003\end{array}$ & $\begin{array}{c}r=.210^{* *} \\
p=.034\end{array}$ \\
\hline & Participación social (4) & $\begin{array}{l}r=.223^{*} \\
p=.024\end{array}$ & $\begin{array}{c}r=.332 * * \\
p=.001\end{array}$ & n.s. & $\begin{array}{c}r=.281 * * \\
\mathrm{p}=.004\end{array}$ & n.s. \\
\hline & Muerte (5) & n.s. & n.s. & $\begin{array}{c}r=.457^{* *} \\
p=.000\end{array}$ & n.s. & n.s. \\
\hline & Morir (6) & n.s. & n.s. & $\begin{array}{c}r=.855^{* *} \\
p=.000\end{array}$ & $\begin{array}{l}r=.199 * \\
p=.045\end{array}$ & n.s. \\
\hline
\end{tabular}

Nota: $* *$ La correlación es significativa al nivel 0.01 (bilateral); *La correlación es significativa al nivel 0.05 (bilaterial); n.s. = no significativa.

\section{Análisis de comparación}

Se hallaron diferencias estadísticamente significativas al comparar las variables de sexo y $\mathrm{CV}$ $(U=.036, p<.05)$, no así entre sexo y AeRAC $(U=.394, p>.05)$. En el caso de los niveles de (mala, regular, buena y muy buena) y AeRAC (mala, regular, buena y muy buena), también se observaron diferencias $(K=12.560, p<.05)$. Estos mismos

Instituto de Investigaciones Psicológicas - Universidad Veracruzana ISSN impreso: 1405-1109 resultados no se apreciaron al comparar los niveles socioeconómicos del AMAI y la CV $(U=.576$, $p>.05)$, ni en los niveles del AeRAC $(U=.387$, $p>.05)$.

\section{DISCUSIÓN}

La presente investigación tuvo como objetivo identificar las diferencias estadísticas y la asociación 
de la calidad de vida y la autoeficacia para realizar actividades cotidianas en una muestra de AM mexicanos. Sociodemográficamente, los hallazgos coinciden con lo reportado por Montes de Oca (2013), quien considera la viudez como el estado civil más frecuente en esta población; luego entonces, este último podría tener un importante efecto en su CV, ya que se ha identificado que vivir en compañía -con la pareja, por ejemplo-permite tener una CV satisfactoria (Garbaccio et al., 2018).

En dicho análisis sociodemográfico se halló que la mayoría de los participantes se encontraba entre los tres niveles más altos del AMAI, haciéndoles posible una estabilidad económica y un mayor acceso a atención médica. De acuerdo con Cardona et al. (2018) y Garbaccio et al. (2018), contar con suficientes ingresos económicos es un factor que permite tener un "buen" nivel de CV; sin embargo, los análisis de comparación demostraron que no había diferencias estadísticamente significativas entre los niveles socioeconómicos del AMAI y los niveles de CV y AVD. Por otro lado, la seguridad social entre los participantes fue de $98.1 \%$, lo cual difiere de lo señalado por Villegas y Montoya (2014), quienes reportaron que los AM padecen una baja seguridad social. Contar con acceso a un seguro médico favorece la atención de las patologías que padecen los AM -que en esta investigación estaban presentes en $82.4 \%$ de los participantes-, puede beneficiar la sensación de control y aumentar la autoeficacia para enfrentar las complicaciones de una enfermedad crónica, motivo por el cual la CV y las AVD podrían no verse afectadas.

La evaluación de los constructos de interés en los participantes identificó que los hombres alcanzan una mejor CV que las mujeres -siendo estadísticamente significativa-, pero no en las AVD, lo que coincide con lo reportado por Soria y Montoya (2017). De acuerdo con Sirgy (2012), se dice que las mujeres suelen presentar mejor $\mathrm{CV}$, pese a lo cual hay una serie de factores que pueden beneficiar la percepción de esta variable en cualquier sexo, tales como la edad, la cultura, el lugar de residencia, el estado civil, el nivel educativo y la religión, por solo mencionar algunos.

Los resultados obtenidos en los niveles de CV y AVD entre ambos sexos difieren de lo encontra-

Instituto de Investigaciones Psicológicas - Universidad Veracruzana ISSN impreso: 1405-1109 do por González y Lima (2017), quienes hallaron mejores CV y AVD en su muestra (69.57 y 67.88\%, respectivamente); por otro lado, coinciden con los resultados de Loredo, Gallegos, Xeque, Palomé y Juárez (2016), que encontraron niveles similares de CV, pero no en cuanto al nivel de independencia y autocuidado referido por los participantes (80.2\% de su muestra era independiente, y $76 \%$ reportó tener buen autocuidado). De igual forma, los presentes resultados de la asociación entre las variables coinciden con los de los autores antes mencionados al encontrar una asociación de magnitud pequeña. Cabe resaltar que en la presente investigación se midió el constructo de autoeficacia para realizar actividades cotidianas, y que en el estudio de Loredo, Gallegos, Xeque, Palomé y Juárez (2016) se evaluó la independencia y el autocuidado, midiéndose la CV mediante el cuestionario SF-36. En consecuencia, los resultados obtenidos deben analizarse bajo la estructura de los instrumentos utilizados: el WHOQoL-OLD y el AeRAC-Breve, ya que su definición conceptual y operacional difiere del SF-36, el índice de Barthel y el cuestionario de capacidades de autocuidado empleado por los autores (Gobbens y Remmen, 2019; Hsieh, Hoffmann, Gustafsson y Lee, 2012; Vanleerberghe, Witte, de Claes, Schalock y Verté, 2017).

La asociación de magnitud moderada estadísticamente significativa entre la tercera dimensión de ambos instrumentos (intimidad en el WHOQOLOLD y sociales en el AeRAC-Breve) resalta el posible efecto que tienen una red de apoyo social y la interacción con los demás sobre la CV y la autoeficacia de los AM para realizar actividades diarias (González, 2010; Granados y Muñoz, 2015; Mendoza y Martínez, 2009). La asociación más alta $(r=.855, p<.001)$ se obtuvo de la sexta dimensión del WHOQOL-OLD - que aborda el temor a morir y a la muerte- con la tercera dimensión del AeRACBreve -que considera la relación con familiares, amigos y vecinos-. Una posible explicación de tales resultados es que las mujeres suelen experimentar un mayor temor a su muerte o a la de un conocido, por lo que son quienes más desean vivir debido a los lazos de afecto que mantienen con otras personas y a la preocupación sobre el futuro de las mismas (Rodríguez, Valderrama y López, 2007). 
Con base en los resultados del presente estudio, es posible concluir lo siguiente: 1) Poco más de la mitad de la muestra de AM obtuvo niveles de bajos a intermedios en su CV, lo que es consistente con lo hallado en estudios previos efectuados en población mexicana. 2) Más de la mitad de los participantes poseía una autoeficacia buena o muy buena para la realización de sus actividades cotidianas, pese a que más de $80 \%$ sufría de al menos una enfermedad crónica, por lo que se resalta la percepción que tienen los adultos mayores de la ejecución exitosa de su actividades, no obstante que presenten complicaciones de salud. 3) Es necesario proseguir el estudio de la relación entre la $\mathrm{CV}$ y autoeficacia en la realización de AVD, así como con los componentes de la capacidad funcional, de tal manera que se formule un modelo teórico sólido que se posibilite el diseño de intervenciones psicológicas destinadas a la población de AM. 4) Por último, se sugiere que futuras investigaciones dispongan de una muestra equivalente para disminuir la probabilidad de resentir amenazas a su validez interna.

Citación: Pérez-Ortiz, A., Becerra-Gálvez, A.L., Hernández-Gálvez, G.A., Estrada-Alvarado, M.C. y Medina-Jiménez, E.A. (2022). Calidad de vida y actividades de la vida diaria en adultos mayores mexicanos. Psicología y Salud, 32(1), 171-179. https:// Doi.org/10.25009/pys.v32i1.2723.

\section{REFERENCIAS}

Asociación Mexicana de Agencias de Inteligencia de Mercado y Opinión (2017). Nivel SocioEconómico AMAI 2018. Ciudad de México: AMAI. Recuperado de http://www.amai.org/nse/wp-content/uploads/2018/04/Nota-Metodolo\%CC\%81gico-NSE2018-v3.pdf.

Bandura, A. (1997). Self-efficacy. The exercise of control. New York: W. H. Freeman and Co.

Cardona A., D, Segura C., Á, Garzón D., M.O. y Salazar Q., L.M. (2018). Condiciones físicas, psicológicas, sociales, emocionales y calidad de vida de la población adulta mayor en el departamento de Antioquia. Papeles de Población, 24, 9-42. Doi: 10.22185/24487147.2018.97.23.

Castillo, D. y Vela, F. (2005). Envejecimiento demográfico en México. Evaluación de los datos censales por edad y sexo, 1970 2000. Papeles de Población, 11(45), 107-141.

Consejo para Prevenir y Eliminar la Discriminación en la Ciudad de México (2016). Personas adultas mayores. Ciudad de México: COPRED: Recuperado de http://data.copred.cdmx.gob.mx/por-la-no-discriminacion/personas-adultas-mayores/.

Estrada, A., Cardona, D., Segura, Á.M., Chavarriaga, L.M., Ordóñez, J. y Osorio, J.J. (2011). Calidad de vida en adultos mayores de Medellín. Biomédica, 31(4), 492-502.

Garbaccio, J.L., Tonaco L., A.B., Estêvão, W.G. y Barcelos, B.J. (2018). Aging and quality of life of elderly people in rural areas. Revista Brasileira de Enfermagem, 71, 724-732. Doi: 10.1590/0034-7167-2017-0149.

Gobbens, R.J.J. y Remmen, R. (2019). The effects of sociodemographic factors on quality of life among people aged 50 years or older are not unequivocal: comparing SF-12, WHOQOL-BREF, and WHOQOL-OLD. Clinical Interventions in Aging, 14, 231-239. Doi: 10.2147/CIA.S189560.

González C., A.L. (2009). Autoeficacia para realizar actividades cotidianas (AERAC) en ancianos mexicanos. En A. L. González-Celis (Ed.): Evaluación en psicogerontología (pp. 47-74). México: El Manual Moderno.

González C., A.L. (2010). Calidad de vida en el adulto mayor. En L. M. Gutiérrez y J. H. Gutiérrez (Eds.): Envejecimiento humano: Una visión transdisciplinaria (pp. 365-378). Recuperado de http://www.geriatria.salud.gob.mx/descargas/publicaciones/envejecimiento_humano.pdf.

González C., A.L. y Gómez B., J. (2013). Quality of life in the elderly: Psychometric properties of the WHOQOL-OLD module in Mexico. Health, 15, 110-116. Doi: 10.4236/health.2013.512A015.

González C., A.L. y Lima, L. (2017). Autoeficacia, percepción de salud y soledad sobre la calidad de vida en adultos mayores. Entreciencias: Diálogos en la Sociedad del Conocimiento, 5, 3-18. Doi: 10.22201/enesl.20078064e.2017.15.62571.

González C., A.L. y Raya L., E. (2017). Generatividad en adultos mayores mexicanos: asociación entre interés y comportamientos generativos. Psicología y Salud, 27(1), 79-88.

González, K.D. (2016). Envejecimiento demográfico en México: análisis comparativo entre las entidades federativas. Ciudad de México: Consejo Nacional de Población.

Granados, M. y Muñoz, D. (2015). Factores que inciden en la calidad de vida de las personas adultas mayores. Enfermería Actual en Costa Rica, 29. Recuperado de https://www.redalyc.org/articulo.oa?id=44839779006. 
Grembowski, D., Patrick, D., Diehr, P., Durham, M., Beresford, S., Kay, E. y Hecht, J. (1993). Self-efficacy and health behavior among older adults. Journal of Health and Social Behavior, 34(2), 89-104. Doi: 10.2307/2137237.

Hsieh, C.L., Hoffmann, T., Gustafsson, L. y Lee, Y.C. (2012). The diverse constructs use of activities of daily living measures in stroke randomized controlled trials in the years 2005-2009. Journal of Rehabilitation Medicine, 44(9), 720-726.

Huenchuan, S. (2013). El cambio de paradigma: la consideración del envejecimiento como un asunto de derechos humanos. En V. Z. Montes de Oca (Ed.): Envejecimiento en América Latina y el Caribe. Enfoques de investigación y docencia de la Red Latinoamericana de Investigación en Envejecimiento (pp. 561-598). Ciudad de México: UNAM.

Instituto Nacional de las Mujeres (2015). Situación de las personas adultas mayores en México. Ciudad de México: INMUJERES. Recuperado de http://cedoc.inmujeres.gob.mx/documentos_download/101243_1.pdf.

Instituto Nacional de las Personas Adultas Mayores (2019). Envejecimiento y vejez. Ciudad de México: INAPAM. Recuperado de https:/www.gob.mx/inapam/es/articulos/envejecimiento-y -vejez?idiom=es.

Lesson, G.W. (2013). The demographics of population ageing in Latin America, the Caribbean and the Iberian Peninsula, 1950, 2050. En V. Z. Montes de Oca (Ed.): Envejecimiento en América Latina y el Caribe. Enfoques de investigación y docencia de la Red Latinoamericana de Investigación en Envejecimiento (pp. 53-74). México: UNAM.

Loredo F., M.T., Gallegos T., R.M., Xeque M., A.S., Palomé V., G. y Juárez L., A. (2016). Nivel de dependencia, autocuidado y calidad de vida del adulto mayor. Enfermería Universitaria, 13, 159-165. Doi: 10.1016/j.reu.2016.05.002.

Martínez V., N., Ibarrola, C., Fernández, A. y Lafita, J. (2017). El concepto de funcionalidad como ejemplo del cambio del modelo nosológico tradicional. Atención Primaria, 50(1), 65. Doi: 10.1016/j.aprim.2017.03.013.

Mendoza N., V.M. y Martínez M., M.L. (2009). Escala de redes de apoyo social para adultos mayores (ERASAM). En A. L. González-Celis (Ed.): Evaluación en psicogerontología (pp. 95-112). México: El Manual Moderno.

Montes de Oca V., Z. (2013). La discriminación hacia la vejez en la ciudad de México: contrastes sociopolíticos y jurídicos a nivel nacional y local. Perspectivas Sociales, 15(1), 47-80.

Organización Mundial de la Salud (1996). La gente y la salud: ¿qué calidad de vida? Ginebra: OMS.

Organización Mundial de la Salud (2015). Informe mundial sobre el envejecimiento y la salud. Ginebra: OMS.

Power, M., Quinn, K., Schmidt, S. and the WHOQOL-OLD Group (2005). Development of the WHOQOL-OLD module. Quality of Life Research, 14, 2197-2214. Doi: 10.1007/s11136-005-7380-9.

Rodríguez, A.F., Valderrama, L. y López, S. (2007). Actitud y miedo a la muerte en adultos mayores. Pensamiento Psicológico, 3(8), 109-120.

Romero D., M. (2007). Actividades de la vida diaria. Anales de Psicología, 23, 264-271. Doi: 10.6018/analesps.

Sirgy, M.J. (2012). Effects of other demographic factors on subjective QOL. En M. J. Sirgy (Ed.): The Psychology of Quality of Life (pp. 95-108). New York: Springer. Doi: 10.1007/978-94-007-4405-9.

Soria R., Z. y Montoya A., B.J. (2017). Envejecimiento y factores asociados a la calidad de vida de los adultos mayores en el Estado de México. Papeles de Población, 23, 59-93. Doi: 10.22185/24487147.2017.93.022.

United Nations Organization (2019). World Population Ageing 2019. New York: UNO. Recuperado de https://www.un.org/en/ development/desa/population/publications/pdf/ageing/WorldPopulationAgeing2019-Highlights.pdf.

Vanleerberghe, P., Witte de, N., Claes, C., Schalock, R.L. y Verté, D. (2017). The quality of life of older people aging in place: a literature review. Quality of Life Research, 26, 2899-2907. Doi: 10.1007/s11136-017-1651-0.

Villegas V., K.G. y Montoya A., B.J. (2014). Condiciones de vida de los adultos mayores de 60 años o más con seguridad social en el Estado de México. Papeles de Población, 20(79), 133-167.

Instituto de Investigaciones Psicológicas - Universidad Veracruzana

ISSN impreso: 1405-1109
Psicología y Salud, Vol. 32, Núm. 1: 171-179, enero-junio de 2022 https://doi.org/10.25009/pys.v32i1.2723 